\title{
Multicultura e interculturalidad: implicaciones de una ausencia en la educación
}

\author{
Fernando Carlos Aguero-Contreras'
}

Cándida Rosa Urquiza-García"

\section{Resumen}

Reflexión teórica desde las perspectivas sociológica - antropológica de la educación, sustentada en los enfoques de la multicultura e interculturalidad. Se parte de varios estudios realizados en la residencia estudiantil de la Universidad de Cienfuegos, al centro - sur de Cuba. Estos han combinados análisis cuantitativos y cualitativos, considerando variables e indicadores socio demográficos, relacionados con la satisfacción en el acceso a la cultura, la salud, las relaciones y dinámicas sociales, mediadas por una estructura social clasista y sociocultural que distingue especificidades en la biografía cultural de individuos y grupos. Se concedió importancia a la significación dada a la residencia como escenario relevante de la vida educacional, las percepciones de las relaciones múltiples y diversas entre los grupos que tienen presencia en ella, así como los niveles de diálogos en los ámbitos académicos, científicos y sociales. Se analizaron varios documentos, y se realizaron observaciones, entrevistas estructuradas y en profundidad. Desde este contexto se muestran las implicaciones del déficit teórico en la gestión formativa en la residencia universitaria, limitando el alcance del proyecto educativo como concreción curricular, para el desarrollo de procesos socializadores más coherentes. Se concluye que la ausencia de estas perspectivas teóricas en la educación superior posibilitaron que la visión política ocupara la primera interpretación del proceso, expresada en la acción solidaria y aun cuando se muestran resultados concretos de importancia en cuanto al número de alumnos graduados, el proceso formativo fue limitado en el diálogo profundo, la socialización cultural para la lograr la unidad en la diversidad.

\section{Palabras clave}

Universidad - Multiculturalidad - Interculturalidad - Residencia estudiantil - Cuba.

I- Universidad de Cienfuegos, Cuba.

Contacto: faguero@ucf.edu.cu

II- Universidad de Cienfuegos, Cuba. 


\title{
Multiculture and interculturality: implications of a lack in education
}

\author{
Fernando Carlos Aguero-Contreras' \\ Cándida Rosa Urquiza-Garcíal
}

\section{Abstract}

This is a theoretical reflection from the sociological and anthropological perspectives of education, sustained in the focuses of multiculture and interculturality. The presentation is based on several studies carried out in the student residence at Universidad de Cienfuegos, in the Center-South of Cuba. They have combined quantitative and qualitative analysis, considering social, demographic variables and indexes related to satisfaction with access to culture, health, social life mediated by social class and sociocultural structure, which distinguishes specificities in the cultural biography of individuals and groups. Importance was attributed to the meanings given to the residence as a relevant space for educational life, the perceptions of the multiple and diverse relationships between the groups in it, as well as the levels of dialogues in the academic, scientific, and social fields. Several documents were analysed, and observations, questionnaires and depth interviews were conducted. From this context, the implications of the theoretical deficit in the educational management of the university residence have emerged. Such deficit limits the educational project in terms of curriculum development aimed at a more coherent socialization process. It is concluded that the lack of theoretical perspectives in higher education led the political view to occupy the first interpretation of the process, expressed in solidarity actions, and even when concrete and important results in the number of graduates are evidenced, the formative process was limited in terms of deep dialogue, cultural socialization to achieve unity among diversity.

\section{Keywords}

University - Multiculturality - Interculturality - Student residence - Cuba.

I- Universidad de Cienfuegos, Cuba.

Contact: faguero@ucf.edu.cu

II- Universidad de Cienfuegos, Cuba. 


\section{Introducción}

Se valoran estudios en la Universidad de Cienfuegos, ubicada al centro sur del archipiélago cubano, con grupos estudiantiles que conviven en la residencia estudiantil universitaria. Allí existe una diversidad sociocultural originada por la procedencia social clasista y geoespacial, acrecentada por la práctica solidaria con organizaciones, gobiernos e instituciones de diversas regiones del mundo. El análisis multicultural da cuenta de la diversidad de intereses, motivaciones, prejuicios entre individuos, facilita caracterizar la comunicación, apreciar las diferencias de clase, grupos, y género. Posibilita visualizar los patrones culturales que portan los individuos procedentes de la socialización primaria, mediada por el contexto histórico-cultural. Por tanto multicultura e interculturalidad como categorías antropológicas- sociológicas aportan al currículo una educación abierta, participativa, crítica, sustentada en una profunda ética hacia las diferencias, asumiendo posiciones emic y etic. Ellas posibilitan asumir una visión dialéctica de las diferencias, para establecerlas como recurso pedagógico, y alcanzar aprendizajes significativos.

Análisis de documentos de archivos de la residencia y la universidad y entrevistas a 56 alumnos mostraron diversidad sociodemográfica, variada procedencia en la estructura socialclasista en nacionales y foráneos. Cuestionarios centrados en satisfacción con la vida en la residencia en años 2004, 2007 y 2011 a 42, 76 y 82 estudiantes respectivamente mostraron resultados favorables, y satisfacción en los accesos a los servicios sociales y derechos democráticos, pero ratificaron prejuicios, mediados por el género, la procedencia urbano-rural y la mayor o menor influencia cultural familiar. Entrevistas semi estructuradas y en profundidad, a veintiséis alumnos en 2004 posibilitaron captar significados conferidos a la residencia universitaria, mostrándola como espacio de: libertad, responsabilidad, aprendizajes sociales y contradicciones. Otras entrevistas a 51 alumnos (2012) verificaron limitaciones en la comunicación, la socialización y consumo cultural en la residencia. Desconocer la multicultura e interculturalidad en la gestión curricular y en el proyecto educativo, permitieron definir como objetivo, argumentar teóricamente la relevancia de esas categorías para el proceso de formación de los alumnos en la residencia estudiantil.

\section{Multicultura e interculturalidad:}

\section{razones de una ausencia}

La sociedad cubana ha sido fruto de transculturaciones continuas, (ORTIZ, 1991) integrando étnicamente africanos, hispánicos, chinos, indígenas y de otras procedencias aunque se ha mostrado recientemente una homogeneidad importante (GUANCHE, 2008). Otros procesos diferenciadores más allá de lo étnico no se han reconocido, lo cual ha tenido implicaciones sociales. El Congreso Nacional de Educación y Cultura de 1970 aunque trascendental para la educación, presentó resultados que no particularizaron en los análisis sociales, desconociendo la multicultura e interculturalidad. No hizo la distinción urbano-rural, (AGUERO, 2006) aun cuando coincidió en el tiempo con un auge de escuelas en el campo para acercar la teoría a la práctica, la escuela con el trabajo y la educación con la vida. Estas perspectivas han estado ausentes en los análisis de la educación.

Las limitaciones en las concepciones de las ciencias sociales y el papel de la cultura, expandidas desde el segundo lustro de la década del sesenta del siglo pasado condujeron a la paralización de un debate científico en torno a los conflictos de clases, al cierre de la carrera de sociología y la renuncia al papel de disciplinas como la antropología y el trabajo social (ESPINA PRIETO et al., 1995). Tal perspectiva coincidió con la expansión de criterios referentes a que el materialismo histórico era suficiente como 
concepción global de las ciencias sociales en el nuevo proyecto social que se construía, (MARTÍNEZ HEREDIA, 2008). Este proceso impuso un lastre en la visión sociológica y antropológica de la educación, cuyos reflejos aún se perciben en el presente.

Tal como se reconoce prosperó una confusión entre pensamiento social y filosofía por la deformación profunda y empobrecedora del marxismo, que primó durante décadas (MARTÍNEZ HEREDIA, 2008). Aun en el presente muchos de los rasgos positivos que tienen hoy el pensamiento y las ciencias sociales en Cuba no trascienden adecuadamente en la formación del estudiantado universitario limitando los procesos de socializadores y el consumo cultural, esencialmente por la falta de debate e intercambios desde la diversidad. Ello determina que la reasunción crítica del marxismo y los beneficios de sus desarrollos son consumidos por minorías, creándose una división entre élites y masas en el consumo de los bienes culturales (MARTÍNEZ, 2008). Esto explica las limitadas aportaciones de la sociología y la antropología a la construcción y desarrollo de las políticas sociales, particularmente en el campo de la educación. Por tanto la naturaleza cuestionadora y crítica del discurso educativo, cedió espacio a posiciones apologéticas.

Algunos de estos vacíos teóricos sociales se comenzaron a cubrir parcialmente en la década de los ochenta, con resultados científicos acerca de la estructura social clasista (CIPS, 1989), los estudios de la familia, sus tendencias y variaciones a nivel de las regiones, y los estudios acerca de la juventud (DOMÍNGUEZ, 1988). Sin embargo no se focalizan las especificidades culturales en la estructura social clasista, la familia, los grupos generacionales o en la juventud como grupo social relevante. Fue desconocido y reproducido como único criterio causal de la multicultura el componente étnico, obviando que es intrínseca a todas las sociedades (JULIANO, 1993).

Las transformaciones en la economía cubana para subvertir los impactos de la debacle del socialismo en Europa y la antigua Unión Soviética, originaron reformas al modelo económico (1990 - 1992), "para sobrevivir unas y promover desarrollo otras”, (LÓPEZ, 1994, p. 67) las cuales, acrecientan la diferenciación social, pero conducen a que las universidades fomenten la cooperación y el intercambio académico internacional. Crecieron el número de especialistas que prestan servicio científicos técnicos en el exterior como los profesionales que comparten sus experiencias y literatura científica de Europa, Canadá y América Latina. Aumentaron los programas de Maestrías y Doctorados, que en el periodo 2001 - 2006, cuadruplican los matriculados, y graduados en el campo de las ciencias sociales (REPÚBLICA DE CUBA, 2008).

La complejización del escenario social cubano no tuvo un reflejo en los análisis educacionales en publicaciones científicas como la Revista de Educación en varios años, (2000 - 2009) pudiendo discernir que aun cuando abordaron temas relevantes, se desconocen los enfoques relacionados con la multicultura e interculturalidad. Las referencias al diagnóstico como necesidad escolar (LÓPEZ MACHÍN, 2009), para conceder al currículo una dimensión científica, los abordajes de la universalización en la educación superior (DE LA CRUZ LÓPEZ, 2008), los enfoques del pensamiento complejo (CHÁVEZ, 2009) no incluyen las perspectivas referidas, lo que muestra más claramente las implicaciones epistemológicas que las ausencias de las perspectivas sociológicas antropológicas, impusieron en la educación.

Un análisis del significado de la educación en Cuba desde la Campaña de Alfabetización en 1961 hasta finalizar el 2009 mostró la dimensión política de todo el proceso (TURNER MARTÍ, 2009) sin referir a sus implicaciones sociológicas o antropológicas. Con similar perspectiva otra valoración de la educación superior, develó que al tiempo que se universalizó, hacia todos los segmentos de la sociedad, incorporaba a estudiantes de las más diversas latitudes. El análisis presentado mostró que, los estudiantes extranjeros 
preparados en Cuba hasta el 2001, de todos los continentes ascendían a 79.778 alumnos de los cuales un 40,3\% fueron preparados en la educación superior y un 59,7\% en la educación (VECINO ALEGRET, 2002). Esta valoración enfatizó la relevancia política, su aporte cultural como experiencia pedagógica pero se limitó a estos términos.

Solo en artículos científicos, conferencias internacionales y cursos presentados por estudiosos procedentes del exterior que participan en los análisis de la educación en Cuba, se hace referencia abierta a la dimensión multicultural de los procesos educativos. Así en el Congreso Internacional de Educación de 1997, en la conferencia magistral impartida por el teólogo Frei Betto, al examinar la relación entre lo que acontece en el mundo y Cuba en el campo de la educación y la vida social, hace múltiples referencias a "la multiculturalidad como factor a considerar en el despliegue de las políticas sociales" (BETTO, 2007, p. 6) para destacar que tal distinción solo es posible de hacer desde la política y no desde el mercado como impuso la norma neoliberal.

Coincidentes con los años noventa del siglo $\mathrm{XX}$ se reflejan tendencias negativas en la sociedad cubana que impactan en la educación superior. Se hace notorio el proceso de blanqueamiento de la universidad cubana en relación a la menor proporción de ingresos de negros y mestizos a la universidad comparado con su real proporción en las estadísticas sociodemográficas del país, (ÁREAS HERRERA, 1995). Se mostró la feminización de la educación superior (ESPINA PRIETO el al., 1995), en referencia a la mayor proporción de mujeres en la mayoría de las carreras universitarias (ESPINA PRIETO, 2008). Estas circunstancias abrieron espacios para tratar aspectos nunca antes examinados, como los relacionados con la marginalidad (PAZ et al., 2001), la desigualdad, la discriminación racial y la pobreza (ESPINA PRIETO, 2000), así como hacer abordajes que permitieran el análisis de las contradicciones de la sociedad cubana (LIMIA DAVID, 1991; ACANDA, 2002), lo que exigió ajustes y giros en la metodología y epistemología (ESPINA PRIETO, 2001) de las ciencias sociales.

El VII Congreso Internacional de Educación Superior celebrado en la Habana (2010) resaltó contenidos multiculturales y experiencias interculturales desde otras instituciones regionales. Estos procesos se relacionaron con la internacionalización de la educación superior y la necesidad de reconocer el contexto multicultural especialmente para el desarrollo del intercambio con países del sur. $\mathrm{Al}$ abogar por la internacionalización solidaria, se enfatizó en el empeño de enfrentar unidos los dilemas de la globalización y los retos del desarrollo asociados a la investigación científica, la certificación de la calidad y la acreditación de saberes. En este encuentro científico se hizo un análisis de la cantidad de cursantes extranjeros en las universidades cubanas en los años 2000 - 2009, como resultado de la colaboración, los que ascendieron a 31343 alumnos a nivel de pregrado, mientras que en el postgrado la cifra ascendió a 8845 profesionales de prácticamente todas las regiones del mundo (VILLAVICENCIO PLASENCIA, 2010).

En los dos congresos internacionales de educación superior al inicio de la segunda década (2012 y 2014) la presencia de los temas relacionados con la multicultura e interculturalidad tuvieron una mayor frecuencia. En el VIII Congreso Internacional de 2012, especialmente en sus talleres dedicados a la internacionalización de la educación superior y sus perspectivas, universidades españolas, norteamericanas y latinoamericanas hicieron referencia a la relevancia de los referidos temas. Se mostró que las universidades tienen con la internacionalización el imperativo de promover la interculturalidad en la formación, (BENNETT MILTON, 2012), lo cual favorece la educación en la diferencia y el pensamiento crítico, (MELÉNDEZ GARCÍA, 2012). No hubo referencia a estos temas desde el ámbito nacional. 
Más intenso aun, fue la presencia de los temas mencionados en el IX Congreso Internacional de Educación Superior de 2014. Se reconocieron las relaciones entre multicultura e interculturalidad con la calidad y pertinencia (NAVA GÓMEZ; CEDILLO MONTESILLO, 2014), el incremento delos compromisosy responsabilidades de los graduados, el imperativo de particularizar las diferencias, especialmente los contenidos étnicos raciales (VILAR DE LIMA; BARBOSA DE SOUZA; GESTERMEIER DO NASCIMENTO, 2014) y garantizar el enfoque comunitario con más realismo (GIL FLORES; GARCÍA LÓPEZ; VERDUZCO GODOY, 2014). Definitivamente se reconoce que el simple cruce cultural al interior de las universidades deviene simple si no se procura un aprendizaje intercultural (SERENA VEGETTI; BENVENUTO, 2014).

Las experiencias de los trabajos presentados en los congresos internacionales de educación superior de La Habana contrastan con la irrelevancia concedida a la naturaleza esencialmente multicultural, que ha caracterizado a la educación superior cubana, en especial su residencia estudiantil y consiguientemente, la limitada visión de este escenario con grandes potencialidades para el despliegue de la interculturalidad en el proceso formativo.

Los análisis realizados aunque no reproducen la perspectiva metodológicas de Fantini, (2009) comparten el criterio de la interculturalidad como habilidad compleja que implica asumir las perspectivas etic y emic, para lo cual será indispensable un conjunto de atributos y valores, como la flexibilidad, la tolerancia, la empatía, unido a habilidades para comunicar, cooperar en tareas de interés mutuo, y habilidad para desarrollar relaciones interpersonales (FANTINI, 2009).

Aunque en el plano internacional creció la importancia concedida por la educación superior a la multicultura e interculturalidad evidenciado en los congresos de educación superior celebrados en Cuba, y en la literatura internacional, en el plano interno no hubo abordajes de estas temáticas.

\section{Sentido y razón de la multicultura e interculturalidad}

Una práctica educacional efectiva debe tener la capacidad reconocer los huecos pedagógicos cuando la institución educativa reconoce escenarios escolares con diferenciaciones sutiles y múltiples, y no flexibiliza su quehacer para reconocer los ajustes imprescindibles (CAMILLERI, 1985). Los análisis aportados por las ciencias sociológicas y antropológicas en referencia al papel de los sentidos, las percepciones, la memoria y la psiquis humana en la formación y los procesos de aprendizajes han sido esenciales. Estas ciencias mostraron relaciones de los procesos lingüísticos, los esquemas de pensamientos, los referentes culturales y los comportamientos con los aprendizajes, la estructura social, los grupos económicos y las subculturas, lo que llevó a cuestionar los intentos de ofrecer recetas pedagógicas únicas en escenarios diversos como socialmente más complejos en estructuras (CAMILLERI, 1985).

La aparición de la multiculturalidad como una respuesta minoritaria al fracaso de la educación compensatoria especialmente en la década del sesenta del siglo XX en Estados Unidos de Norteamérica (MCCARTHY, 1999), muestra los tres enfoques principales que han prevalecido: la comprensión, la competencia y la emancipación cultural. El primero sustentado en el relativismo cultural, resalta la validez de las expresiones culturales, como base para eliminar los prejuicios, mostrando tempranamente su ineficacia al desconocer las implicaciones educativas de la biografía cultural.

El segundo caso da primacía al pluralismo cultural en el currículo, a partir de la tolerancia y el enriquecimiento mutuo de la totalidad, buscando desde lo multicultural la comprensión y la conciencia de la diferencia, privilegia el idioma y la cultura de la pluralidad, manteniendo la autoctonía de los grupos para desde una interacción transcultural, reducir los antagonismos. El tercer enfoque aboga por la emancipación cultural y la 
reconstrucción social como mejora social para las minorias, lo que destaca dos principios esenciales: el respeto a cada lengua, etnia y biografía cultural, consignando que el déficit de los alumnos se originan en los maestros, y el reconocimiento de que cada biografía cultural recoge una historia de privaciones (MCCARTHY, 1999). Aunque estas perspectivas tomaron auge en los setenta y ochenta del siglo XX concentraban la mayor responsabilidad en las aulas desconociendo el peso de la desigualdad estructural y de las relaciones de poder.

Hacia finales del siglo XX se enfatiza la tendencia a reconocer las políticas de inclusión en los programas educacionales, para el fomento de la perspectiva multicultural de la educación. Se ha destacado (ZOLLERS; RAMANATHAN; MOONSET, 1999) que el proceso organizativo, visto como los valores y creencias compartidos que interactúan con una organización de personas, estructuras, y sistemas de control, generan normas de comportamiento, propician un lenguaje rico y diverso lo cual resulta un factor esencial para el éxito de los procesos inclusivos. Este análisis sin embargo no reconoce el peso de la estructura jurídica y el acceso a los derechos ciudadanos reales para acceder a los beneficios de las políticas sociales.

La multiculturalidad devino en la transición al siglo XXI un tema de especial relevancia bajo los efectos de la globalización, las tensiones migracionales y en consecuencia un elemento de elevada trascendencia política (WITHTOL DE WENDEN, 2003). La internacionalización de la educación superior generó procesos estresores de aculturación asociados a las barreras lingüísticas, limitaciones en los procesos de aprendizajes, discriminación, soledad $\mathrm{y}$ otros problemas prácticos asociados a ambientes generales (SMITH; KHAWAJA, 2011). Estas circunstancias generan posiciones en los individuos con un espectro amplio de estrategias donde se pueden incluir la integración, la marginación, la asimilación o la separación. Se ha demostrado que los niveles de interrelaciones e integración entre estudiantes pueden generar niveles importantes de satisfacción
(HENDRICKSON; ROSEN; AUNE, 2011). De igual forma en los escenarios educativos donde los principios del colectivismo grupal e institucional son más elevados, la orientación hacia el futuro y la búsqueda de la igualdad de género son más reales, los resultados del aprendizaje, la formación multicultural y las competencias interculturales son más evidentes (JOY; KOLB, 2009).

La multiculturalidad se articula a las concepciones de comunidades (JANSEN, 2004) y ciudadanía (GURHARPAL, 2003). Su utilidad facilita la descripción de la diversidad cultural como elemento que legitima la incorporación de grupos sociales a la gestión de gobierno y como política pública aboga por la unidad en la diversidad (BELKER; LEILDÉ, 2003).

La multiculturalidad se emplea como elemento descriptivo de la diversidad social, clasista y étnica, aunque también destaca otros indicadores como la procedencia, los rasgos epiteliales y los propios elementos de la cultura. Se hace evidente la estrecha relación entre los procesos culturales y las diferenciaciones socioculturales que se construyen en los grupos humanos a partir de sus prácticas históricas, sociales, etnoculturales y económicas. Tal como se afırma "las culturas raramente, si no jamás, son totalizadoras y monolíticas" (MOHAN RA0, 2000, p. 21) pues en toda sociedad con un mínimo de especialización, diferenciación, jerarquización de estatus y poder, las oportunidades y las motivaciones varían según el grupo social.

La historia reconoce que las diferencias étnicas y sociales han sido manipuladas y mal tratadas no solo en la práctica política sino desde las ciencias en general y desde esta en la educación, (SINNREICH, 2006) lo que explica como las deformaciones que la modernidad impuso en la fragmentación de saberes impactaron directamente a la educación. Resulta especialmente complejo las aportaciones que se derivan de las vicisitudes entre política, cultura y ciencia (KUMAR; WELZ, 2001).

Con estas circunstancias se articulan las políticas culturales, pues marcan pautas 
esenciales en la democratización, mostrando definitivamente si existen o no fracturas entre las necesidades y la satisfacción con el consumo cultural en la diversidad social y cultural. Desde este ámbito, la multiculturalidad se convierte en un indicador cualitativo para apreciar si las diferencias en el consumo cultural y el acceso al desarrollo de las políticas sociales en general, se convierte en una conjugación armónica de posibilidades iguales para todos.

Esto plantea a las universidades la necesidad trabajar valores, gustos y modelos de pensamiento críticos, de distinguir el valor de la unidad en la diversidad, requiriendo para el desarrollo de la formación tres principios esenciales: la articulación de la teoría con la práctica, la educación desde la instrucción, acompañada de una activa labor científica cultural para descolonizar el pensamiento y la subjetividad humana, con prácticas educativas desfetichizantes y desenajenantes, generadoras una conciencia crítica, principios y contenidos coincidentes con la esencia de la educación intercultural.

La trascendencia de la interculturalidad como elemento relevante en el quehacer comunitario y educativo se define por la capacidad para describir las culturas diversas y promover el diálogo intercultural. Sin embargo estos análisis concitan la atención a la biografía cultural de los grupos humanos, abogan por la justicia social, los derechos humanos democráticamente establecidos, al reconocimiento cabal y la validez de la diversidad social, clasista y cultural en la sociedad, como muestran estudios en América Latina en la educación superior. Estos destacan el factor clasista (LÓPEZ; SICHRA, 2006), para ir más allá del bilingüismo indígena (LÓPEZ; SICHRA, 2008), logrando una visión ciudadana inclusiva, participativa aunque no exenta de conflictos, (DEJAEGHERE, 2009). Asumen los procesos de la multicultura e interculturalidad como elementos consustanciales de la sociedad civil, (MATOS, 2009), reconociendo las tensiones y diversidad de concepciones
(FERRÃO CANDAU, 2010) aun cuando se expanda la educación (GALLEGUILLOS; CATANI, 2011), y el papel de los sujetos resulte esencial en medio de la relación de saber-poder buscando sociedades no homogeneizantes (CARVALHO, 2012). Implica no desconocer el papel de las familias y las influencias de las tecnologías de la información y las comunicaciones en los procesos socializadores (TAMAYO; RODRÍGUEZ; CARMONA, 2014). Estas perspectivas resultan imprescindibles para asumir los inalienables derechos de preservar la diferencia y la diversidad, como riqueza mayor en la vida contemporánea.

Lainterculturalidad toma relevancia como principio curricular en tanto hace referencia de manera genérica al campo de las relaciones entre culturas diferentes (MATOS, 2009) y por tanto no depende de las características de alumnos o profesores sino a la capacidad institucional para asumir estos procesos desde la participación, el auto reconocimiento, el respeto mutuo y la capacidad para distinguir la validez de las múltiples maneras de asumir, interpretar y actuar en escenarios diversos. Implica el rechazo al acto educativo como proceso de aculturación y subyace en esta perspectiva el reconocimiento de la validez de múltiples saberes. Por tanto la interculturalidad busca construir relaciones equitativas y de valoración mutua, lo cual suele expresarse sintéticamente con la fórmula interculturalidad con equidad (MATOS, 2009) considerando los derechos humanos democráticamente establecidos. Se infiere por tanto la dialéctica entre la multicultura y la interculturalidad y por tanto se enfatiza la necesidad de educar la interculturalidad (STEPHAN; STEPHAN, 2013).

\section{Algunas implicaciones prácticas de las ausencias teóricas}

La educación superior en Cienfuegos en su trayectoria pasó de ser un Instituto Superior Técnico (1979) a la condición de Universidad (1996), alcanzando para el 2006 un total de 8115 
graduados y de ellos 3867 mujeres. Ha formado profesionales en especialidades de ingenierías, ciencias económicas, cultura física, las ciencias pedagógicas, y varias ciencias sociales totalizando doce carreras. Todas las universidades cubanas siempre tuvieron una residencia estudiantil para una parte de sus estudiantes.

Las residencias estudiantiles son las áreas universitarias, (pueden ubicarse dentro o fuera del recinto universitario) que dan asiento a personas, que desde diversas procedencias geográficas, arriban a ellas para desarrollar estudios superiores. Toman particular connotación desde la etapa medieval aunque alcanzan una dimensión cultural más clara con el advenimiento del capitalismo. En el marco de esa sociedad mostró su naturaleza exclusiva al acrecentar los costos de la educación superior. Para países ubicados en la periferia del capitalismo, las residencias universitarias muestran más claramente su naturaleza clasista (URQUIZA GARCÍA; AGÜERO CONTRERAS, 2010).

El acceso a las residencias universitarias cubanas se produce de forma libre y sin costo. Se acogen en ellas a personas de procedencias geográficas, económicas, socioclasistas y culturales muy diversas. La vida en la residencia concede el derecho a múltiples servicios de amplio beneficio social-cultural: accesos a radio, TV, prensa escrita, e internet, servicios médicosanitarios, estomatológicos, y la acción de los programas de prevención de enfermedades, las programaciones de vacunaciones, así como la atención sistemática de especialistas en trabajo educativo, psicólogos, para la orientación educativa (URQUIZA GARCÍA, 2003). Los equipos multidisciplinarios que laboran en las residencias cuentan con la asistencia de otros especialistas y de los profesores de las propias instituciones universitarias. Así la concepción de la residencia es insertada a la de formación de los estudiantes y como espacio que se integra a la labor extensionista y cultural de la universidad (MOLINA PRENDES, 2005), resultando un proceso atinado, coherente e imprescindible.

Desde esta perspectiva la concreción y contextualización curricular en la universidad (LÓPEZ MACHÍN, 2009) expresada en el proyecto educativo, concede especial significado a la residencia universitaria. El proyecto educativo, sustentado en el diagnóstico científico, plasma metas, necesidades básicas y complementarias en el proceso de formación. Por ello tienen presencia en él, elementos cognitivos, éticos, estéticos, lúdicos y profesionales, que en los diferentes momentos y espacios de la vida universitarias (HERNÁNDEZ LÓPEZ, 1999), los estudiantes han determinado asumir, en consensos grupales y de conjunto con sus profesores. Tienen significación en el proyecto educativo además aspectos relacionados con la salud, el deporte, la cultura y las competencias profesionales que en los cursos académicos deben ir alcanzando.

La orientación de salud en lo relacionado a la sexualidad responsable, la prevención del SIDA, la estética y la higiene en la vida cotidiana, la educación formal y las relaciones de pareja, como la prevención del alcoholismo y la violencia, adquieren también particular relevancia. Todo el proceso en la residencia universitaria se apoya, en el caso de la experiencia que sirve de base al presente análisis, en la autodirección estudiantil, caracterizada por su independencia y autonomía (URQUIZA GARCÍA, 2003). La identificación de estos contenidos culturales en la residencia, a diferencia de posiciones tecnocrática enriquecen la relación universidad sociedad, refuerza la formación cultural (DE LA CRUZ LÓPEZ, 2008), lo que deviene premisa de la integralidad que se busca.

La tabla $n$. 1 , ofrece una idea de la dimensión foránea en la residencia, elemento que la identificó por primera vez como escenario multicultural. 
Tabla I- Procedencia según regiones de estudiantes extranjeros

\begin{tabular}{|c|c|c|c|c|c|c|c|c|c|}
\hline \multirow[b]{2}{*}{ N. } & \multirow{2}{*}{$\begin{array}{l}\text { Curso Escolar/ } \\
\text { Regiones de procedencia. }\end{array}$} & \multicolumn{2}{|c|}{$1997-1998$} & \multicolumn{2}{|c|}{$1998-1999$} & \multicolumn{2}{|c|}{$2000-2001$} & \multicolumn{2}{|c|}{$2001-2002$} \\
\hline & & $\begin{array}{l}\text { Formación } \\
\text { Pregrado } \\
\end{array}$ & $\begin{array}{l}\text { Formación } \\
\text { Postgrado }\end{array}$ & $\begin{array}{l}\text { Formación } \\
\text { Pregrado } \\
\end{array}$ & $\begin{array}{l}\text { Formación } \\
\text { Postgrado }\end{array}$ & $\begin{array}{l}\text { Formación } \\
\text { Pregrado }\end{array}$ & $\begin{array}{l}\text { Formación } \\
\text { Postgrado } \\
\end{array}$ & $\begin{array}{c}\text { Formación } \\
\text { Pregrado }\end{array}$ & $\begin{array}{l}\text { Formación } \\
\text { Postgrado } \\
\end{array}$ \\
\hline & Caribe Insular menos Cuba & 120 & - & 293 & 5 & 254 & 20 & 103 & 70 \\
\hline 2. & América Central & 0 & - & 5 & 0 & 22 & 1 & 0 & 4 \\
\hline 3. & América del Sur & 0 & - & 0 & 0 & 0 & 4 & 9 & 7 \\
\hline 4. & África & 3 & - & 6 & 19 & 1 & 48 & 5 & 46 \\
\hline 5. & Oriente Medio & 4 & - & 0 & 0 & 0 & 0 & 30 & 0 \\
\hline 6. & Asia & 0 & - & 2 & 0 & 0 & 0 & 10 & 0 \\
\hline 7. & América del Norte & 1 & - & 0 & 0 & 0 & 0 & 0 & 0 \\
\hline & Total General & $128^{1}$ & - & $306^{2}$ & 24 & $277^{3}$ & 73 & 157 & $127^{4}$ \\
\hline
\end{tabular}

Fuente: Archivo residencia estudiantil Universidad de Cienfuegos. Elaboración propia.

1. Este grupo incluyó 120 estudiantes del área del Caribe, de los cuales: 40 proceden de Granada, 30 de Saint Vicent, 21 de Santa Lucia, 19 de Dominica, 3 de Haití, 3 de Saint Kitts y Nevis, y 3 de Monserrat. Se añaden a estos, cuatro del Medio Oriente, 3 de Siria y uno de Palestina. Tres proceden de África: 1 de Guinea Bissau, 1 de Ghana y 1 de Mauritania. Se añade un estudiante de Canadá. De este grupo 78 son mujeres y 50 son hombres.

2. De este grupo concentra la mayor proporción al área del Caribe: 23 son de Saint Kitts y Nevis, 47 de Hait́́, 2 de Monserrat, 35 de Saint Vicent, 25 de Antigua y Barbudas, 79 de Santa Lucia, 31 de Dominica, 2 de Guadalupe, 5 de Surinam, 17 de Jamaica, 26 de Barbados, 1 uno de Bahamas. De Centro América solo tienen presencia 5 de Belice y 6 de África, 5 de Guinea Konacry y 1 de Mali.

3. Este grupo incluyó 254 jóvenes estudiantes del Caribe, distribuidos de la siguiente manera: 133 de Bahamas, 108 de Jamaica, 13 de Santa Lucia. Se incluyen 22 estudiantes de América Central, procedentes de Guatemala, Panamá, Belice, Nicaragua

4. Como se puede apreciar en estos años creció continuamente el número de estudiantes a nivel del postgrado, lo que incrementan la diversidad: tiene presencia estudiantes de Canadá, Suecia, Holanda, Dinamarca, e Italia, que viene a estudiar esencialmente aspectos relacionados con la lengua española y la cultura cubana. Tienen presencia estudiantes que no se recogen en las cifras del pregrado procedentes de Estados Unidos de la Universidad de Tacoma, única Universidad Norteamericana autorizada por aquel gobierno a que sus estudiantes tuviesen estadías de 90 días en la Universidad de Cienfuegos. Se distinguen, a nivel de postgrado grupos Canadienses que llegan para estudiar procesos diversos relacionados con el desarrollo sostenible.

La diversidad se acrecentó en la medida que la experiencia del trabajo y las relaciones se acrecentaron con los diferentes países de procedencia de los alumnos. Para el curso académico 2007 - 2008 la distribución de estudiantes por áreas de procedencia correspondió a 37 países: 39 de África (21,8\%), 31 de Asia (17,3\%), 23 de América Latina (continental) (12,8\%) y 86 del Caribe Insular (48,0\%), (ARCHIVOS UCF, 1979 - 2014). Por tanto de los 738 alumnos en la residencia universitaria 179 son extranjeros los que representan un 24,25\% de la totalidad. A nivel de las diferentes carreras que se estudian en ese momento en la Universidad presenta la siguiente distribución vista en la tabla 2.

Aunque la cantidad de alumnos cubanos en la residencia se mantuvo en los años y cursos escolares subsiguientes, la cuantía de extranjeros tuvo reducciones. Así en el 2008 -2009 hubo 126 alumnos foráneos, en el curso 2009 - 2010, 99 alumnos, y en el curso escolar $2011-2012$ ascendieron a 66 alumnos procedentes de: Caribe
Insular, África, América Continental, y Asia. Para el curso 2012 - 2013 arribaron 61 alumnos de: Caribe Insular, África, Oriente Medio y Asia (ARCHIVOS UCF, 1979 - 2014).

A las características derivadas por la presencia de extranjeros se añaden algunas características de los estudiantes cubanos que destacan por primera vez y de forma más objetiva la referida naturaleza multicultural del escenario que representa la residencia universitaria. Entre el grupo de cubanos se constató que un 72\% se reconocen blancos, un 18\% mestizos y un 10\% negros. Se apreció mayor proporción de mujeres en la casi totalidad de los cursos, el 65\% de los alumnos residen en zonas más urbanizadas y el 35\% a zonas menos urbanizadas o rurales, pertenecientes a cinco de las provincias cubanas, más de diecisiete municipios y unos sesenta asentamientos poblacionales (ARCHIVOS UCF, 1979-2014). Al mismo tiempo se pudo constatar que la totalidad de los alumnos en la residencia universitaria, se relacionaban con ecosistemas costeros, cuencas 
Tabla 2- Estudiantes cubanos y extranjeros en la residencia universitaria según carreras.

\begin{tabular}{|c|c|c|}
\hline \multirow{2}{*}{ Carreras que se estudian } & \multicolumn{2}{|c|}{ Estudiantes en la residencia universitaria } \\
\hline & Extranjeros & Cubanos \\
\hline Ingeniería Informática & 41 & 158 \\
\hline Ingeniería Mecánica & 23 & 53 \\
\hline Ingeniería Industrial & 19 & 37 \\
\hline Licenciatura en Contabilidad. & 24 & 36 \\
\hline Licenciatura en Economía & 34 & 81 \\
\hline Licenciatura en Estudios socioculturales & 1 & 29 \\
\hline Licenciatura en Lengua Inglesa & 1 & 19 \\
\hline Licenciatura en Derecho & 6 & 67 \\
\hline Licenciatura en Cultura Física & 0 & 37 \\
\hline Licenciatura en Historia & 0 & 12 \\
\hline $\begin{array}{c}\text { Curso preparatorio en Lengua Española para iniciar la educación } \\
\text { superior en diferentes carreras }\end{array}$ & 30 & 0 \\
\hline Total & 179 & 559 \\
\hline
\end{tabular}

Fuente: Elaboración propia. Archivo Universidad de Cienfuegos (UCF), 1979 -2014 (curso 2007 - 2008).

hidrográficas, y montañas. No reconocer estas diferenciaciones muestra huecos pedagógicos referidos por Camillieri (1985) limitando el proceso de formación.

Las actividades económicas que prevalecen en las zonas de procedencia de los alumnos cubanos en la residencia y que condicionan sus culturas, se relacionan con la agricultura y otros procesos agropecuarios como la producción azucarera, el tabaco, el café, la ganadería. Alcanza relevancia también actividades relacionadas con el turismo, la industria, la pesca y más aun con el comercio y los servicios sociales como educación, salud. El $47 \%$ sostiene algún tipo de creencia religiosa, encontrándose expresiones de la casi totalidad de variantes del protestantismo, los cultos sincréticos de origen africano y las relacionadas con la iglesia católica. Un 53\% expresó no identificarse con una creencia particular. Desde el punto de vista social clasista se identificó la procedencia de los alumnos de la residencia universitaria en un $38,6 \%$, con procedencia de intelectuales, técnicos y administrativos, un $26,7 \%$ de procedencia obrera, un 34,7\% de una amplia gama de trabajadores agrícolas y agropecuarios, en correspondencia con la re estratificación de la sociedad cubana consecuencia de las transformaciones económicas que confırman la variabilidad sociocultural y particularmente multicultural del escenario nacional. Tal variabilidad genera una multiplicidad de expresiones en la subjetividad (MOHAN RA0, 2000), y dan cuenta de trazas socioculturales relevantes más allá de lo étnico para consignar relevancia a lo socioeconómico y clasista (JULIANO, 1993).

Por lo tanto la residencia estudiantil universitaria presenta un cruce de culturas aun antes del arribo de estudiantes extranjeros. Las entrevistas muestran que los alumnos procedentes de áreas menos urbanizadas $\mathrm{y}$ de estructuras sociales de menor rango económico presentan niveles culturales más bajos, limitaciones académicas, en el consumo cultural y menor desenvolvimiento social y participativo. La disfuncionalidad familiar muestra tendencias hacia la conflictividad. Los conocimientos recíprocos son generales y limitados. Se han apreciado discordias en la percepción que construyen de las atenciones que reciben cubanos y extranjeros 
en la residencia. Puntos de conflictos fueron reconocidos en relación a las prácticas religiosas, entre cubanos, latinoamericanos y árabes; en las relaciones entre africanos de la región del Magreb y región subsahariana. Las prácticas alimentarias entre los procedentes de Asia y los de otras regiones, en las concepciones amorosas y la sexualidad responsable de africanos y latinoamericanos y caribeños, entre las concepciones de la historia y los modelos del desarrollo entre caribeños y latinoamericanos y cubanos. Las diferencias se visualizan con mayor intensidad mediadas por los factores clasistas que por los étnicos, raciales, regionales, si bien en determinados momentos matizan estos procesos.

El cuadro que se presenta seguidamente, recoge elementos de la plataforma de trabajo del perfil profesional y metas del proyecto educativo en varias carreras como de la residencia universitaria. El análisis destaca valores, principios y rasgos en la formación, distingue una convergencia importante con las metas de la residencia estudiantil universitaria. No reconoce la multicultura en la residencia y ni considera el principio de la interculturalidad.

Cuadro 1- Metas del proyecto educativo, modelo profesional y la residencia estudiantil universitaria.

\begin{tabular}{|c|c|c|c|}
\hline \multirow[b]{2}{*}{ Carreras } & \multicolumn{2}{|c|}{$\begin{array}{l}\text { Valores coincidentes en el modelo profesional de carreras } \\
\text { seleccionadas }\end{array}$} & \multirow{2}{*}{$\begin{array}{l}\text { Metas del trabajo en la } \\
\text { residencia estudiantil } \\
\text { universitaria }\end{array}$} \\
\hline & $\begin{array}{l}\text { Valores ideo políticos } \\
\text { - Solidaridad } \\
\text { - Internacionalismo }\end{array}$ & $\begin{array}{c}\text { Principales } \\
\text { rasgos profesionales } \\
\text { del modelo }\end{array}$ & \\
\hline $\begin{array}{l}\text { Licenciatura en estudios } \\
\text { socioculturales } \\
\text { Ingeniería mecánica }\end{array}$ & $\begin{array}{l}\text { Valores culturales } \\
\text { - Compañerismo } \\
\text { - Formación holista } \\
\text { - Comunicación } \\
\text { - Estéticos } \\
\text { - Amor al estudio }\end{array}$ & $\begin{array}{l}\text { - Integral } \\
\text { - Crítico } \\
\text { - Innovador } \\
\text { - Revolucionario } \\
\text { - Independencia } \\
\text { - Dominio idioma extranjero } \\
\text { - Comprometido } \\
\text { - Amante de la paz y justicia } \\
\text { - Creativo } \\
\text { - Culto } \\
\text { - Investigativo } \\
\text { - Trabajar en equipo. }\end{array}$ & $\begin{array}{l}\text { - Orden } \\
\text { - Amor al estudio } \\
\text { - Práctica deportiva } \\
\text { - Limpieza } \\
\text { - Belleza } \\
\text { - Honradez } \\
\text { - Respeto } \\
\text { - Amistad } \\
\text { - Sinceridad } \\
\text { - Deber } \\
\text { - Comunicar } \\
\text { - Solidaridad } \\
\text { - Convivencia colectiva }\end{array}$ \\
\hline
\end{tabular}

Fuente. Elaboración propia. Residencia Universitaria, Universidad de Cienfuegos 2002. Síntesis de los Protocolos de trabajo de los Consejos de Carreras de la Universidad de Cienfuegos. Cursos académicos $2001-2010$.

Como se aprecia en el cuadro anterior $\mathrm{n}^{0} 1$; resulta indispensable que a diferencia de lo establecido por McCarthy (1999) se reconozcan las potencialidades de la diversidad multicultural como base para el diálogo intercultural. El escenario multicultural se 
debe utilizar como refuerzo de la formación ciudadana, conjugando la dialéctica de lo global y lo local, para alcanzar la unidad en la diversidad (BELKER; LEILDÉ, 2003). Los valores presentes en los proyectos educativos culturales, políticos y éticos, observados desde los rasgos que se definen en el perfil profesional y las metas de la residencia universitaria coinciden con la perspectiva de Fantini (2009) ofreciendo la posibilidad de colocar el enfoque intercultural en el proyecto educativo (STEPHAN; STEPHAN, 2013), pues desde esta plataforma existe la posibilidad de trabajar la interculturalidad con equidad (MATOS, 2009).

Con el objetivo de verificar las implicaciones de estos procesos se realizaron entrevistas a un grupo de estudiantes cubanos y extranjeros, con la intención de apreciar la magnitud e intensidad del diálogo, pues en correspondencia con las perspectiva teóricas tratadas, procesos formativos más ricos e intensos debían lograrse siempre que la comunicación alumno-alumno alcance una fluidez mayor en los principales momentos del proceso de enseñanza aprendizaje.

Tabla 3- Diálogo auto reconocido por estudiantes en la residencia universitaria (\%).

\begin{tabular}{|c|c|c|c|c|}
\hline \multirow{2}{*}{ N. } & \multirow{2}{*}{$\begin{array}{c}\text { Escenario del dialogo } \\
\qquad N=51 \\
\text { [26 extranjeros y } 35 \text { cubanos] }\end{array}$} & \multicolumn{3}{|c|}{$\begin{array}{l}\text { Nivel del dialogo entre estudiantes cubanos y extranjeros en la } \\
\text { residencia universitaria. (\%). }\end{array}$} \\
\hline & & Alto & Medio & Bajo \\
\hline 1) & $\begin{array}{l}\text { Académico: aulas, actividad de estudio, realización de } \\
\text { proyectos científicos, tareas docentes. }\end{array}$ & 25 & 40 & 35 \\
\hline 2) & $\begin{array}{l}\text { Extracurricular: actividades en la producción, los } \\
\text { servicios, la investigación. }\end{array}$ & 10 & 48 & 42 \\
\hline 3) & Vida Social: cultural recreativo, lúdicas, etc. & 30 & 45 & 25 \\
\hline
\end{tabular}

Fuente: Elaboración propia. Entrevistas realizada por los autores, Mayo - Septiembre de 2012.

La tabla anterior muestra la tendencia al desarrollo del diálogo limitado entre los estudiantes cubanos y extranjeros en los ámbitos académico-curricular y extra curricular, como en la vida social, lo cual se aprecia como una debilidad del proyecto educativo y que permite entender las respuestas de estos alumnos a temas más profundo de la vida de los pueblos que representan.

En diálogos informales y en las entrevistas realizadas, muchos estudiantes foráneos, mostraron desconocimientos o criterios muy generales relacionados con la realidad cubana y del proyecto educativo, como concreción curricular construida desde los propios estudiantes y profesores. Similar diálogo con estudiantes cubanos mostró desconocimiento de aspectos esenciales de la vida social, económica y cultural de pueblos con cuyos alumnos convivieron periodos de tiempo prolongados. Solo un diálogo de carácter intercultural puede abrir al enriquecimiento mutuo desde las diferentes culturas, ampliando las posibilidades de la formación y de ahondar la unidad en la diversidad. Esta dimensión ética del proceso contribuye al cambio de mentalidad colectiva (FRANK-OLAF, 2003), pero resulta clave generar normas de comportamiento, que propicien un lenguaje compartido, base de los procesos inclusivos (ZOLLERS; RAMANATHAN; MOONSET, 1999).

Al finalizar cada curso académico los estudiantes reciben una evaluación desde la residencia universitaria de manera integral dando cuenta de aspectos relacionados con la formación general. Un análisis secuencial 
de los procesos evaluativos en la residencia durante varios cursos académicos ha permitido apreciar como tendencia que entre un $18 \%$ y un $22 \%$ se evalúan de excelente, entre un 75\% y un $80 \%$ se evalúa de bien y que solo entre un $2 \%$ y un $4 \%$ se evalúa con la categoría de deficiente o mal. Entre los indicadores principales de esta evaluación se incluyen, la disciplina, la responsabilidad social mostrada, cumplimiento de sus deberes, la higiene, la salud, y la dedicación al estudio. Por lo tanto el resultado de estos procesos se discute individual y colectivamente a nivel de los grupos en la residencia y se integra como una evaluación general junto con los resultados académicos. Los déficits mencionados se reproducen en estos actos de evaluación.

\section{Conclusiones}

Aun cuando en la región latinoamericana y en el mundo se reconocen la relevancia de la perspectiva multiculturaly dela interculturalidad para el despliegue de los procesos de formación en la educación superior, en Cuba, la concepción de la solidaridad como categoría política concentró la visión del proceso. Esto no limitó las contribuciones hechas en miles de profesionales formados de muchas regiones del mundo, pero inhibió procesos relevantes para lograr mayor efectividad en la formación.
Superada la desigualdad estructural al contar con el acceso a las políticas sociales y socializadas las relaciones de poder, al disponer la residencia universitaria en la Universidad de Cienfuegos, de la autodirección estudiantil, el ambiente político facilita el despliegue de la multicultura e interculturalidad, pero los déficits teóricos en las ciencias sociales y en particular en la sociología y la antropología de la educación han impedido su reconocimiento y consecuentemente han limitado su aplicación para el proceso formativo.

Las ausencias referidas han determinado el reconocimiento formal y parcial de la biografía cultural de los estudiantes, sin distinguir las implicaciones curriculares, manteniendo las interacciones transculturales, basadas en la tolerancia desde la solidaridad. La plataforma ético - jurídica, garantía de real acceso a derechos democráticos y políticas sociales desde proyecto educativo en varias carreras universitarias muestra un sistema de acciones y valores que coinciden con las bases de la multicultura e interculturalidad pero se perciben socialmente como procesos aculturales , en tanto la ausencia del diálogo profundo y de consumo cultural desde la rica diversidad sociocultural presente y las construcciones colectivas no afloran coherentemente, como rasgos de la internacionalización de la educación superior en la era global.

\section{Referencias}

ACANDA, Jorge Luis. Sociedad civil y hegemonía. La Habana: Centro de Investigación y Desarrollo de la Cultura Cubana Juan Marinello, 2002.

AGUERO CONTRERAS, Fernando Carlos. Sociedad, cultura y currículum escolar. Berlín: Verlag Berlin WVB, 2006.

ARCHIVOS UNIVERSIDAD DE CIENFUEGOS. Registros de estudiantes en la residencia universitaria: cursos académicos 1979 2014. Cienfuegos: Universitaria, 2014.

ÁREAS HERRERA, Héctor. La comunidad y su estudio: personalidad, educación y salud. Madrid: Pueblo y Educación, 1995.

BELKER, Simon; LEILDÉ, Anne. Is multiculturalism a workable policy in south Africa? International Journal of Multicultural Societies, París, v. 5, n. 2, p. 121-136, 2003.

BENNETT, Milton J. Turning cross-cultural contact into intercultural learning. In: TALLER INTERNACIONAL LA EDUCACIÓN SUPERIOR 
Y SUS PERSPECTIVAS, 11., 2012, La Habana. [y] CONGRESO INTERNACIONAL DE EDUCACIÓN SUPERIOR, 8., 2012, La Habana. IX Taller... La Habana: [s. n.], 2012. CD 1.

BETTO, Frei. Neoliberalismo y educación. Conferencia magistral en el Congreso Internacional de Educación de 2007. Revista Cubana de Educación, La Habana, v. 2, n. 9, p. 2-9. 2007.

CAMILLERI, Carmel. Antropología cultural y educación: preparado para la Oficina Internacional de Educación. París: Unesco, 1985.

CARVALHO, Rosângela Tenório de. 0 discurso curricular intercultural na educação de jovens e adultos e a produção de subjetividades. Educação e Pesquisa, São Paulo, v. 38, n. 1, p. 47-61, ene./mar. 2012.

CIPS - CENTRO DE INVESTIGACIONES PSICOLÓGICAS Y SOCIOLÓGICAS. Estudio sociológico sobre la estructura socio clasista de Cuba. La Habana: Academia, 1989.

CHÁVEZ, Armando. Enseñanza de la filosofía. Revista Cubana de Educación, La Habana, n. 127, p. 7-19, 2009.

DE LA CRUZ LÓPEZ, Luiz Miguel. Hacer realidad una esencia. Revista Cubana de Educación, La Habana, n. 125, p. 24-31, 2008.

DEJAEGHERE, Joan G. Critical citizenship education for multicultural societies. Inter-American Journal of Education for Democracy, Washington, v. 2, n. 2, p. 222-236. 2009.

DOMÍNGUEZ, Maria Isabel. Algunos criterios metodológicos para la investigación de la juventud. Revista Cubana de Ciencias Sociales, La Habana, v. 4, n. 17, p. 99-116, 1988.

ESPINA PRIETO, Mayra P. El giro epistemológico y sus derivaciones para el pensamiento social emancipatorio: informe de investigación. La Habana: Centro de Investigaciones Psicológicas y Sociológicas, 2001.

ESPINA PRIETO, Mayra P. Expresiones territoriales del proceso de estratificación: resultado final. La Habana: Centro de Investigaciones Psicológicas y Sociológicas, 2000.

ESPINA PRIETO, Mayra P. La comprensión de la desigualdad: 1-46. La Habana: Centro de Investigación Psicológicas y Sociológicas, 2008.

ESPINA PRIETO, Mayra P. Tropiezos y oportunidades de la sociología cubana. Revista Temas, La Habana, v. 1, p. 36-49, mayo 1995.

ESPINA PRIETO, Mayra P. et al. Impacto socio estructural del reajuste económico. La Habana: Centro de Investigación Psicológicas y Sociológicas, 1995.

FANTINI, Alvino E. Assessing intercultural competence: issues and tools. In: DEARDORFF, Darla (Ed.). Handbook of intercultural comptence. London: Sage Publishing, 2009. p. 457-476.

FRANK-OLAF, Radtke. Multiculturalism in Germany: Iocal management of immigrants' social inclusion. International Journal of Multicultural Societies, París, v. 5, n. 1, p. 55-76, 2003.

FERRÃO CANDAU, Vera Maria. Educación intercultural en América Latina: distintas concepciones y tensiones actuales. Estudios pedagógicos, Valdivia, v. 36, n. 2, p. 333-342. 2010.

GALLEGUILLOS, Tatiana Gabriela B.; CATANI, Afrânio Mendes. Avaliação da educação superior no Brasil e a expansão da educação superior em enfermagem. Educação e Pesquisa, São Paulo, v. 37, n. 4, p. 843-860, dic. 2011.

GIL FLORES, Hugo C.; GARCÍA LÓPEZ, Glária S.; VERDUZCO GODOY, Arturo. Interculturalidad: una necesidad formativa para el docente de hoy. In: TALLER INTERNACIONAL DE FORMACIÓN DE PROFESIONALES DE EDUCACIÓN 5., 2014, La Habana. [y] CONGRESO INTERNACIONAL DE EDUCACION SUPERIOR, 8., 2014, La Habana. V Taller... La Habana, Palacio de Convenciones, 2014.

GUANCHE, J. Componentes étnicos de la nación cubana. Edición revisada y ampliada. La Habana: Fundación Fernando Ortíz, 2008.

GURHARPAL, S. Multiculturalism in contemporary Britian: reflection on the Leicester Model. International Journal of Multicultural Societies, París, v. 5, n. 1, p. 40-54. 2003. 
HENDRICKSON, B; ROSEN, D; AUNE, R. K. An analysis of friendship networks, social connectedness, homesickness, and satisfaction levels of international students. International Journal of Intercultural Relations, Florida, v. 35, n. 3, p. 281-295, mayo 2011.

HERNÁNDEZ LÓPEZ, Irma de Las Nieves. El enfoque cultural integral en las universidades cubanas de cara al siglo XXI. Revista Cubana de Educación Superior, La Habana, v. 21, n. 2, p. 81-92. 1999.

JANSEN, EPW. The influence of the curriculum organization on study progress in higher education. Higher Education, Netherlands, n. 47, p. 411-435. 2004.

JOY, Simy; KOLB, David A. Are there cultural differences in learning style? International Journal of Intercultural Relations, Florida, v. 33, n. 1, p. 69-85, enero. 2009.

JULIANO, Dolores. Educación intercultural: escuela y minorías étnicas. Barcelona: [s. n.], 1993. (Cuadernos de antropología).

KUMAR, Anand; WELZ, Frank. Culture in the world-system: an interview with immanuel wallerstein. Journal of World System Research, Pittsburgh, v. 7, n. 2, p. 221-231, 2001.

LIMIA DAVID, Miguel. Las contradicciones de la sociedad cubana contemporánea. Revista Cubana de Ciencias Sociales, La Habana, v. 25, p. 88-108, 1991.

LÓPEZ, Delia Luisa. Período especial y democracia en Cuba. Cuadernos Africa América Latina - Revista de Análisis Sur Norte para una cooperación Solidaria, Madrid, v. 94, n. 164, p. 53-76. 1994.

LÓPEZ, Luis Enrique.Cultural diversity, multilingualism and indigenous education in Latin America. In: GARCIA, Ofélia; SkUTNABBKANGAS, Tove; TORRES-GUZMAN, Maria E. (Ed.). Imagining multilingual schools: language in education and globalization (linguistic diversity and language rights). Great Britain: Cromwell, 2006. p. 238-261.

LÓPEZ, Luis Enrique; SICHRA, Inge. Intercultural bilingual education among indigenous peoples in Latin America. In: HORNBERGER, Nancy (Ed.). Encyclopedia of language and education. Washington: Springer, 2008. p. 1732-1746.

LÓPEZ MACHÍN, Ramon. El diagnóstico en la escuela. Revista Cubana de Educación, La Habana, n. 126, p. 24-31. 2009.

MARTÍNEZ HEREDIA, Fernando. El ejercicio de pensar. Panamá: Ruth Casa Editorial, 2008.

MATOS, Daniel. Instituciones interculturales de educación superior en América Latina: procesos de construcción, logros, innovaciones y desafíos. Caracas: IESALC: Unesco, 2009.

McCARTHY, Cameron. Enfoques multiculturales de la desigualdad racial en Estados Unidos. In: FERNÁNDEZ ENGUITA, Mariano (Ed.). Sociología de la educación: lecturas básicas y textos de apoyo. Barcelona: Ariel, 1999. p. 221-241.

MELÉNDEZ GARCÍA, Victor A. Educación intercultural: un modelo educativo para la gestión favorable de la diferencia y el desarrollo crítico de la ciudadanía en tiempos de globalización. In: TALLER INTERNACIONAL LA EDUCACIÓN SUPERIOR Y SUS PERSPECTIVAS, 11., 2012, La Habana. [y] CONGRESO INTERNACIONAL DE EDUCACIÓN SUPERIOR, 8., 2012, La Habana. XI Taller... La Habana: MES, 2012.

MOHAN RA0, J. Cultura y desarrollo económico. México: UNAM, 2000.

MOLINA PRENDES, Norma. Modelo de formación cultural en las universidades: una experiencia cubana. Revista Cubana de Educación Superior, La Habana, v. 25, n. 2, p. 93-100. 2005.

NAVA GÓMEZ, Guadalupe Nancy; CEDILLO MONTESILLO, José Luis. Condiciones para la internacionalización de la Universidad Pública. In: TALLER INTERNACIONALIZACIÓN DE LA EDUCACIÓN SUPERIOR, 7., 2014, La Habana. [y] CONGRESO INTERNACIONAL DE EDUCACIÓN SUPERIOR, 9., 2014, La Habana. VII Taller... La Habana: [s.n.], 2014.

ORTIZ, Fernando. Estudios etnosociológicos. La Habana: Pensamiento Cubano, 1991.

PAZ, JuanValdés et al. Entendemos la marginalidad? Revista Temas, La Habana, n. 27, p. 69-96. 2001.

REPÚBLICA de Ccuba. Oficina Nacional de Estatística - ONE. Inicio del curso 2007-2008 y resumen del curso escolar 2006- 
2007. La Habana: ONE, 2008.

SERENA VEGGETTI, Maria Serena; BENVENUTO, Guido. Inter-university cooperation and "social" education: multicultural frame and joint curricula. In: TALLER INTERNACIONALIZACIÓN DE LA EDUCACIÓN SUPERIOR, 7., 2014, La Habana. [y] CONGRESO INTERNACIONAL DE EDUCACIÓN SUPERIOR, 9., 2014, La Habana. VII Taller... La Habana: [s.n.], 2014.

SINNREICH, Helene J. Using the past to confront the present: teaching minority history in present - day Poland. Electronic Magazine of Multicultural Education, París, v. 8, n. 2, p. 1-7. 2006.

SMITH, Rachel A.; KHAWAJA, Nigar G. A review of the acculturation experiences of international students. International Journal of Intercultural Relations, Florida, v. 35, n. 6, p. 699-713, nov. 2011.

STEPHAN, Walter G; STEPHAN, Cookie White. Designing intercultural education and training programs: an evidence-based approach. International Journal of Intercultural Relations, Florida, v. 37, n. 3, p. 277-286, mayo 2013.

TAMAYO, Dora Liliana 0.; RODRÍGUEZ, Farley Johanna C.; CARMONA, Norma Darío M. Construcción del bienestar juvenil en las actuales dinámicas de socialización. Revista Colombiana de Ciencias Sociales, Bogotá, v. 5, n. 1, p. 77-98, mayo 2014.

TURNER MARTÍ, Lidia Esther. Inicio de una epopeya: la educación en la revolución. Revista Cubana de Educación, La Habana, n. 128, p. 1-24, 2009.

URQUIZA GARCÍA, Cándida Rosa; AGUERO CONTRERAS, Fernando Carlos. Universidad y multiculturalidad: una interpretación teórica. Revista Universidad y Sociedad, Cienfuegos, v. 2, n. 2, p. 1-8, 2010.

URQUIZA GARCÍA, Cándida Rosa. Universidad y multiculturalidad: una interpretación teórica. In: CONFERENCIA INTERNACIONAL, 2. 2003, La Habana. La obra de Carlos Marx y los desafíos del Siglo XXI. II Conferencia... La Habana: [s. n.], 2003. Disponible en: $<$ http://www.nodo50.org/cubasigloXXl>. Acesso en: 4 oct. 2006.

VECINO ALEGRET, Fernando. La educación superior cubano en la búsqueda de la excelencia. Revista Cubana de Educación Superior, La Habana, v. 22, n. 1, p. 18-36, 2002.

VILAR DE LIMA, Marinalva; BARBOSA DE SOUZA, Antonio Clarindo; GESTERMEIER DO NASCIMENTO, Celso. Relações étnicoraciais: formação de professores e responsabilidade da universidade brasileira. In: TALLER INTERNACIONALIZACIÓN DE LA EDUCACIÓN SUPPERIOR, 11., 2014, La Habana. [y] CONGRESO INTERNACIONAL DE EDUCACIÓN SUPERIOR, 9., 2014, La Habana. XI Taller... La Habana: [s.n.], 2014. CD 1.

VILLAVICENCIO PLASENCIA, Maria Victoria. Evolución y perspectivas de los servicios académicos internacionales en el ministerio de educación superior. In: CONGRESO INTERNACIONAL DE EDUCACIÓN SUPERIOR, 7., 2010, La Habana. VII Congreso... La Habana: [s. n.], 2010.

WITHTOL DE WENDEN, Catherine. Multiculturalism in France. International Journal of Multicultural Societies, París, v. 5, n. 1, p. $77-88.2003$.

ZOLLERS, Nancy J; RAMANATHAN, Arun K.; MOONSET, Yu. The relationship between school culture and inclusion: how an inclusive culture supports inclusive education. International Journal of Qualitative Studies in Education, Londres, v. 12, n. 2, p. 157-174. 1999.

Recibido en: 23.02.2015.

Aprobado en: 27.05.2015.

Fernando Carlos Aguero-Contreras es licenciado en educación, historia y ciencias sociales (1979). Diplomado historia de Cuba, Universidad de la Habana (1987). Master en educación, Universidad de Cienfuegos (1996). Ph. D. ciencias de la educación, Universidad de Oviedo España (2002). Postdoctoral en Universidad Muenster, Alemania (2006). Profesor de educación superior 1979-2014.

Cándida Rosa Urquiza-García es licenciada en educación, historia y ciencias sociales (1983) Instituto Superior Pedagógico Félix Varela, Villa Clara. Master en educación, Universidad de Cienfuegos (2002). Especialista en trabajo educativo, residencia universitaria, Universidad de Cienfuegos 1997-2014. 